1992;ㅁ: 15-16). The finding of subtypes of neuroanatomic changes supports the belief that autism is a heterogeneous disorder.

\title{
TOURETTE SYNDROME
}

\section{FREQUENCY, FAMILY AND SOCIAL ASPECTS OF TS}

Results of a prospective, longitudinal study of 21 children recruited at age $21 / 2$ to $31 / 2$ years without tics but with a first-degree relative with Tourette syndrome (TS) are reported from the Department of Psychology and Child Study Center, Yale University, New Haven, CT. All subjects were evaluated annually and for 2 to 4 years. Among these high risk children, 24\% had developed TS, $9 \%$ chronic tics, and $9 \%$ transient tics. Obsessive-compulsive symptoms occurred in 19\%, and obsessive-compulsive disorder in $5 \%$. Other diagnoses included attention deficit disorder, speech problems, and anxiety disorder in $24 \%$. Children in this sample demonstrated an increased risk for tic disorders as well as other psychiatric disorders. Family functioning, independent of parental psychopathology, was associated with attentiondeficit and anxiety disorders, decreased adaptive and increased maladaptive behaviors, and lower self-esteem but not tics or learning disorders. (CarterAS et al. A prospective longitudinal study of Gilles de la Tourette's syndrome. I Am

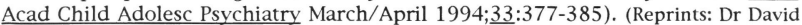
L Pauls, Child Study Center, Yale University School of Medicine, 230 S Frontage Road, New Haven, CT 06510).

COMMENT. An autosomal dominant mode of transmission for TS is suggested by the rates of tic disorders observed. Stressors in family functioning play a role in comorbid disorders such as anxiety and attentional difficulties. The authors advise family, cognitive-behavioral, and interpersonal therapies to address the social-emotional difficulties that often accompany TS.

Clonazepam was a useful adjunctive treatment for tics in children with comorbid ADHD studied at the Children's Hospital, Boston (Steingard RJ et al. IAm Acad Child Adolesc Psychiatry March/April 1994;33:394).

\section{CONGENITAL DEVELOPMENTAL DISORDERS}

\section{SEPTO-OPTIC DYSPLASIA AND DIABETES INSIPIDUS}

The clinical and endocrinological findings in 24 children with septooptic dysplasia and/or agenesis of the corpus callosum are described with reference to posterior pituitary function in a report from the Institute of Child Health and The Hospital for Sick Children, London, UK. Congenital optic nerve hypoplasia, absent septum pellucidum, and pituitary deficiency, characteristic of the complete syndrome of septo-optic dysplasia, were present in 8 children, and 13 had incomplete forms. Five had agenesis of the corpus callosum. Growth hormone insufficiency was found in $20(83 \%)$. Nine $(38 \%)$ had diabetes insipidus, often complicated by hypernatremia. Management of fluid balance was difficult, even with vasopressin treatments, because of blindness, developmental delay, impairment of the sense of thirst, and dependence on the parents for food and water intake. (Masera N, Grant DB et al. Diabetes insipidus with impaired osmotic regulation in septo-optic dysplasia and agenesis of the corpus callosum. Arch Dis Child Jan 1994;70:51-53). (Respond: Dr DB Grant, The Hospital for Sick Children, Great Ormond Street, London WC1N 3JH, England). 